\title{
Action-dependent perceptual invariants: From ecological to sensorimotor approaches
}

\author{
Matteo Mossio* \\ IHPST \\ CNRS/Université Paris I \\ 13, rue du Four \\ 75006 Paris \\ France
}

\author{
Dario Taraborelli \\ Department of Psychology \\ University College London \\ Gower Street \\ London WC1E 6BT \\ United Kingdom
}

\begin{abstract}
Ecological and sensorimotor theories of perception build on the notion of actiondependent invariants as the basic structures underlying perceptual capacities. In this paper we contrast the assumptions these theories make on the nature of perceptual information modulated by action. By focusing on the question, how movement specifies perceptual information, we show that ecological and sensorimotor theories endorse substantially different views about the role of action in perception. In particular we argue that ecological invariants are characterized with reference to transformations produced in the sensory array by movement: such invariants are transformationspecific but do not imply motor specificity. In contrast, sensorimotor theories assume that perceptual invariants are intrinsically tied to specific movements. We show that this difference leads to different empirical predictions and we submit that the distinction between motor dependence and motor specificity needs further clarification in order to provide a more constrained account of action/perception relations.
\end{abstract}

\section{Keywords}

action; perception; self-motion; invariants; sensorimotor theories; ecological psychology; enaction

\footnotetext{
"Acknowledgments. The ideas discussed in this paper have been presented at different stages of development over the last years. We wish to thank David Philipona, Nivedita Gangopadhyay, Roberto Casati, as well as participants in the Enactive Workshop (Paris, 2005), the Perception, Action and Consciousness Conference (Bristol, 2007), the Interactivist Summer Institute (Paris, 2007), the 33 ${ }^{\text {rd }}$ SPP Annual Conference (Toronto, 2007) for valuable feedback on earlier versions of this paper. Dario Taraborelli was partly supported by a grant from the ENACTIVE Network of Excellence (IST-2002002114). Both authors contributed equally to this work. Email: matteo.mossio@ens.fr - Fax : +33 144 278647
} 


\section{Blurring the boundary between action and perception}

The idea that action and perception are not two independent cognitive domains and that perception is constitutively shaped by action has been an important research trend in the last decades. The study of active perception, or those mechanisms that allow organisms to negotiate perceptual problems by processing sensory stimulation on the basis of action, has become a thriving area of investigation in neuroscientific, psychological and computational research on perception, witness the number of reviews and theoretical analyses that have addressed this trend (Thelen \& Smith, 1994; Port \& Van Gelder, 1995; Hurley, 1998; Berthoz, 2000; Findlay \& Gilchrist 2003).

The idea of perception as action-dependent has been particularly emphasized by motor theories of perception, i.e. those approaches claiming that perceptual content depends in an essential way on the joint contribution of sensory and motor determinations (Sheerer, 1984). According to motor theories, perceptual systems are able to discriminate between reafference (sensory input resulting from self-motion) and exafference (sensory input produced by external events) in virtue of their relation to efference (internal information elicited by self-motion). Perceptual systems receive at the same time reafference and efferent copies generated by a given movement and such information is used to perceive a specific action as self-initiated. Over time, the organism learns to establish correlations (supposedly stable and systematic for a given organism in a given environment) between reafference and efference (Gallistel, 1980). The capacity to discriminate between exafference and reafference underlies, according to motor theories of perception, several perceptual distinctions (e.g. self-motion vs. motion in or of the environment) and constancies (e.g. position constancy, see Stark \& Bridgeman, 1983; Duhamel et al., 1992).

Motor theories of perception represent a liberal view of action/perception relations, to the extent that they admit that perceptual capacities can depend on sensorimotor relations on top of purely sensory information (Hurley, 2001). They are mildly liberal, though, insofar as they limit the contribution of action to perception to those perceptual processes that underlie the control of motor behavior. This is consistent with the established view that part of the human perceptual system, geared towards action control and spatial behavior, draws on dedicated processes that are functionally independent from those targeted at perceptual categorization and recognition (Milner \& Goodale, 1995; Jacob \& Jeannerod 2003). But can actiondependent perceptual processing extend beyond what seems to be its natural domain, i.e. perception for motor control and spatial behavior? Can action modulate a broader range of perceptual processes than mainstream perceptual theories assume? Does the perception of properties of the environment (independent on the agent's body configuration or movement) rely on action-dependent information? This is a much stronger claim of action dependence in perception and an issue we aim to tackle in this paper.

A number of research programs have taken a more radical stance on action/perception relations, claiming that action is pervasive in the functioning of perceptual systems. The role of action in perception, they suggest, extends beyond processes targeted at the control of motor behavior and some aspects of perceptual 
performance intrinsically depend on the contribution of action.

Claiming that perception is intrinsically active, inseparable from action or even-as some have argued-reducible to a form of action (Noë 2004), is a controversial hypothesis raising different orders of questions.

At a conceptual level, this hypothesis questions the traditional distinction between perception and action as independent cognitive domains. A lively debate has addressed this claim, and several lines of criticism have been leveled against the claim that perception cannot occur without action (Jacob, 2006; Prinz, 2006). Not surprisingly, more attention has been paid to the theoretical implications of these radical approaches than to the consequences of implementing a radical approach as an empirical research program.

From an empirical perspective, radical approaches on action/perception relations raise an interesting, often understated question. If part of perception is actiondependent, what processes and structures can be invoked to explain how perceptual systems parse and select sensory information on the basis of action? The answer that these theories seem to suggest is that perceptual systems process action-dependent perceptual invariants, or patterns of perceptual information that are intrinsically action-dependent.

In this paper our aim is not to address the question whether action and perception can be considered as mutually independent or to argue in favor or against radical approaches. The goal of our analysis is to articulate the very idea that perception may rely on action-dependent invariants and to contrast the way in which such a notion is characterized in these radical approaches. We propose that an analysis of this notion is a more promising testbed to assess the prospects of radical approaches to action/perception as genuine empirical programs than a generic criticism of some of their (possibly flawed) background assumptions.

\subsection{Radical approaches to action/perception relations}

The most prominent theories that take a radical view of the contribution of action to perceptual processes are those that belong to the ecological approach to perception (Gibson, 1979; Cutting, 1986; Reed, 1996). The ecological approach emphasizes the constitutively active nature of perceptual abilities, and the fact that perceptually relevant information is revealed by active interaction of the observer with the environment. A review of the ecological tradition is beyond the scope of the present analysis. Here we focus on ecological theories as a case of radical view on action/perception relations in order to analyze the notion of perceptual information they endorse.

The contrast class that we consider in this paper is a family of research programs that, although not organized into a unified theoretical framework, share a strong interactivist assumption about the nature of perception. We refer to this class of programs as the sensorimotor approach to perception. The sensorimotor approach puts a strong emphasis on the fact that the basic structures underpinning perceptual skills are couplings between an organism's movements and co-occurring changes in sensory stimulation. In this sense, sensorimotor approaches are a radicalization of classical motor theories of perception insofar as they emphasize the pervasiveness of information about self-initiated movement in perceptual processes beyond those 
targeted at the control of motor behavior.

Although this assumption is not yet articulated into a full-fledged empirical paradigm (such as the ecological approach to perception), a long tradition supports the idea that sensorimotor couplings are key to the understanding of perceptual phenomena. The first explicit acknowledgement of the role of sensorimotor invariants to understand perception can be traced back to Helmholtz, who suggested that:

[w] hen we perceive before us the objects distributed in space, this perception is the acknowledgement of a lawlike connection between our movements and the therewith occurring sensations [...]. What we perceive directly is only this law (Helmholtz, 1878/1977, p.138-139)

Sensorimotricity is a core notion in enactive theories, which take cognition as a capacity resulting from the self-organization of living systems and from the constraints imposed on this activity by the interaction with the external environment (Järvilehto, 1999; Varela, 1979; Maturana \& Varela, 1980; Varela et al., 1991). In the field of neurophysiology, some authors defended the idea that perception reduces to the ability of encoding patterns of covariation between motor patterns and cooccurring sensory stimulation (MacKay 1962, 1987). Building on inspiration that can be traced back to Poincaré (1902), sensorimotricity as the ability to process systematic couplings between sensory and motor information has also been regarded as constitutive to the acquisition of space perception (Philipona et al. 2003; Wolff, 2004). A sensorimotor hypothesis has been recently applied to the study of color categorization, showing that several psychophysical aspects of color perception can be predicted by looking at how invariants in properties of reflecting surfaces are encoded by the perceptual system of an agent actively exploring its environment (Philipona \& O'Regan, 2006). Finally, and more controversially, sensorimotricity has also been proposed as a key to the explanation of the phenomenal character of visual experience and visual consciousness (O'Regan \& Noë, 2001).

Ecological and sensorimotor approaches have often been conflated or considered variations on the same theory (Scholl \& Simons, 2001; Pylyshyn, 2001) insofar as they share an important number of background assumptions, in particular:

A. The claim that many traditional problems in perceptual theory not taking into account the contribution of action (e.g. stimulus disambiguation, inverse optics problems, perceptual binding), are ill-posed;

B. The idea that perceptually relevant patterns of the sensory stimulation are those selected through motion and that static sensory patterns (e.g. properties of the retinal image) are irrelevant to functionally characterize perception;

C. The claim that perceiving does not require possessing detailed internal representations of the external environment;

D. The focus on the intrinsically active nature of perception and the role of the action-perception loops;

These similarities have often been taken as arguments in support of a substantial theoretical continuity between ecological and sensorimotor approaches as opposed to mainstream perceptual theories. ${ }^{1}$ Defendants of a sensorimotor approach have

\footnotetext{
${ }^{1}$ See the debate hosted at http://www.interdisciplines.org/enaction
} 
described their divergence from Gibsonian theories as a mere matter of "explanatory focus" and denied any major theoretical discontinuity between the two approaches (Noë, 2002):

In adopting this view we invoke the role of action and the importance of extracting invariants, and so we are indebted to Gibson. But we harness these ideas for quite different explanatory and theoretical purposes. In other words, whereas Gibson stresses the use of sensorimotor invariants as sources of information, we are stressing the idea that sensorimotor invariants are part of what constitute sensations and perceptual content. We show that Gibson's idea can go farther than Gibson pushed it (O'Regan \& Noë, 2001, p. 1019).

Similarly, defendants of the ecological approach to perception tend to present their theories as an articulation of the enactive approach to perception, fully compatible with a sensorimotor approach (Stoffregen \& Bardy, 2004; Stoffregen et al., 2006). In spite of the large number of shared background assumptions, the continuity between these approaches has been challenged by other authors (Varela, 1991; Hurley, 2001).

What this debate has failed to appreciate is the difference in the specific constraints that these theories put on perceptual information modulated by action. The thesis we defend in the present work is that of a substantial divergence between ecological and sensorimotor approaches to perception: this divergence may not be explicit at a broad theoretical level, but-we argue-underpins specific predictions made by these theories on how action modulates perception.

Our argument is two-folded. On the one hand, we will contrast ecological and sensorimotor approaches to perception by looking at the way in which they characterize the notion action-dependent information. On the other hand, we will argue that, because of their different characterization of perceptual invariants, these approaches make different empirical predictions about movement-mediated perceptual skills and about perceptual information and processes underpinning such skills. We conclude that this distinction between different notions of invariant is crucial to frame any empirical research program on the functional role action plays in perception.

\section{Framing the concept of action-dependent invariants}

The radical nature of ecological and sensorimotor approaches to perception does not simply consist in the claim that perception must be studied by looking at the interaction between a goal-directed, active organism and its environment. ${ }^{2} \mathrm{~A}$ much stronger claim they make is that information grounding perceptual abilities is itself action-dependent. In this sense, referring to modulations of perception through action requires formulating explicit hypotheses on how movement affects perceptual information. In this section, we review the characterizations of the notion of actiondependent invariants that can be found in the ecological and sensorimotor literature and we show to what extent they diverge.

\subsection{Ecological invariants}

\footnotetext{
${ }^{2}$ See section 2.4 below.
} 
An informal characterization of the notion of a perceptual invariant can be found in J. Gibson's seminal work (Gibson, 1959; 1960). By providing a plethora of examples of perceptual structures that can be extracted in action/perception couplings, Gibson offers a sort of extensional characterization of what an action-dependent perceptual invariant may be. The lack of an explicit characterization of what constitutes action-dependent perceptual information has been one of the most criticized aspects of Gibson's theory of perception (Fodor \& Pylyshyn, 1981) and later defendants of the ecological approach to perception have tried to spell out this notion in more precise terms.

A more accurate characterization can be found in Michaels \& Carello (1981) who provide a number of definitions of perceptual invariants. In particular, they distinguish between structural invariants and transformational invariants. On the one hand, structural invariants are defined as those properties of the sensory stimulation that remain constant through motor interaction, even though other properties may vary: as such they determine classes of equivalence that allow distinct perceptual objects to be regarded as the same under this respect. On the other hand, transformational invariants can be characterized as "modes of change" of perceptual objects, i.e. invariant dynamics of sensory stimulation produced by specific transformations on these objects. We might rephrase this by saying that structural invariants are those properties that allow perceptual systems to parse structural components of the environment, whereas transformational invariants allow perceptual systems to detect and track dynamic regularities to which structural components obey.

The distinction between structural and transformational invariants has been criticized by other exponents of the ecological approach (see for instance Cutting, cit., p. 67) ${ }^{3}$. However, a common feature of all these characterizations, that remains faithful to a Gibsonian view, is the idea that invariants are patterns in sensory information that are revealed when an organism engages in motor interaction with the environment, or:

structures that remain invariant despite certain transformations caused by the animal and that therefore might serve to specify persisting environmental resources (Reed, cit., p. 48).

Let us try to put this notion of invariant in the context of the general assumptions of ecological theories of perception. The ecological approach defends the idea that there is a nomological relation between specific states of the agent-environment system and invariant properties of the proximal stimulation they produce on the sensory organs of the perceiver. It is in virtue of this nomological relation-described by the laws of ecological optics, acoustics, haptics... (Gibson, 1979) — that invariants "specify" or carry reliable information about the states of the agent-environment system they refer to. This relation of specification in virtue of ecological laws is the tenet of ecological accounts of perception:

\footnotetext{
${ }^{3}$ Cutting (cit.) proposes the following characterization of an optical invariant: "To be an optic invariant, all information about an object or event must be present in the optic array, measurable at a particular place and time, and valid to all places and times. Thus the invariant is a constant mapping from the proximal image and the distant stimulus, where relations between image (or eye) and stimulus are not fixed" (p. 75).
} 
[i]n ecological optics we typically assume that the information (the invariant) specifies the object or event perceived; that is, we pick up (process) the invariant, and as a result perceive the object properties specified (Cutting, cit., p. 71)

Ecological theories assume that perceptual systems are geared to these proximal invariants insofar as their "pickup" enables the perception of the states of the agentenvironment system. ${ }^{4}$ More precisely, the ecological approach postulates that perceptual systems rely on information provided by so-called "ambient energetic arrays" (Gibson, 1979). By hypothesis, these energetic arrays provide information that is rich enough to specify all relevant properties of the agent-environment system and to perceptually control behavior. ${ }^{5}$ In particular, the ambient array contains multimodal sensory invariants that the agent can rely upon to perceive its body configuration and movements (proprioception), the relation between itself and the environment, for instance in order to determine its position or motion direction (exproprioception), as well as to perceive properties of the external environment (exteroception).

All of these proximal invariants are made available through motor interaction. Since a transformation is needed to reveal the invariant, the availability of perceptual information necessarily requires action, as a condition to submit the sensory stimulation to the appropriate transformations. In this hypothesis lies one of the radical claims ecological theories make with respect to motor action: action is a necessary requirement to obtain perceptually relevant information, and no perceptual ability can occur if invariants specified by action are not available. To clarify the notion of perceptual invariant in the ecological approach, let us review two classical examples.

A first classical example of perceptual invariant revealed through motor transformations is the so-called cross ratio, a visual invariant specifying rigidity. ${ }^{6}$

\section{[INSERT FIGURE 1]}

Figure 1: The geometric definition of cross ratio.

Two fundamental properties of the cross ratio need to be emphasized. First, cross ratios are invariant under all rotations and translations of line L2, translations of line L1 and of point X. Second, it can be proved that cross ratio invariance is preserved whatever the shape of the projection surface (L1) is. The invariance of cross ratio

4 See for instance Lee (1976) in the case of vision and Turvey (1996) for the dynamic touch.

${ }^{5}$ As Warren (2006b) characterize it, "[ i]nformation consists of patterns of stimulation at the receptors that are specific to the ecological state of affairs and are therefore useful in controlling action" (p.367).

${ }^{6}$ As Cutting explains it: Let A, B, C, D be four points on the same straight line (L1). Let X be a point not on that line, and connect all point to $\mathrm{X}$. This creates the new lines of which AX, BX, CX and DX are segments. Let line L2 intersect these new lines at points A', B', C' and D'. Projective geometry tells us that the cross ratios of segments bounded by the points ABCD and A'B'C'D' are the same. In particular, the following segments lengths form the following equal ratios:

$\left(\mathrm{AD} \_\mathrm{BC}\right) /\left(\mathrm{AC} \_\mathrm{BD}\right)=\left(\mathrm{A}^{\prime} \mathrm{D}_{-} \_\mathrm{B} \mathrm{C}^{\prime}\right) /\left(\mathrm{A}^{\prime} \mathrm{C}^{\prime} \_\mathrm{B} \mathrm{D}^{\prime}\right)$

This cross ratio - the product of the longest segment $\overline{A D}$ and the inner segment $\mathrm{BC}$ divided by the product of the segments connecting non-adjacent exterior and interior pairs of points (AC and BD) is invariant under any projection to any point not aligned with $\mathrm{A}$ through $\mathrm{D}$ (Cutting, cit., p. 81). 
under several transformations affecting its geometrical projection makes it a good candidate - from an ecological perspective-as a perceptual invariant. Cross ratios can be taken as information that perceptually specifies object rigidity in our environment. ${ }^{7}$ In this sense, cross ratio is a paradigmatic example of a geometric invariant that remains unchanged throughout transformation in the proximal sensory stimulation of an observer and that bears a reliable informative relation with a property of the distal environment.

A second paradigmatic example of invariant addressed in the ecological literature is motion parallax, i.e. the optical pattern produced by the relative movement of an observer with respect to objects in the visual environment (Gibson, 1950). When an observer moves in space, the resulting displacement of the point of fixation generates different motion of pairs of points in its visual stimulation due to the different distance of distal objects from the fixation point.

\section{[INSERT FIGURE 2]}

Figure 2: Motion parallax. As the eye moves from left to right, closer points move faster than further points

Motion parallax can be analyzed in terms of several differential invariants: divergence, curl and deformation (Koenderink 1975, 1986). ${ }^{8}$ It has been shown that these invariants provide reliable perceptual information about the spatial structure of the stimulus, and provide in particular reliable information for depth perception. Accordingly, a large number of empirical studies have explored humans' capacity to perceive depth (including objects three-dimensional shape) by relying uniquely on motion parallax, a process called structure-from-motion (Rogers \& Graham, 1979; Ono et al. 1986; Steinbach et al. 1991; Ujike \& Ono, 2001; Nawrot, 2003). It has been shown in particular that humans can use motion parallax to perceive depth in passive conditions involving object motion (the "kinetic effect", see Wallach \& O'Connell, 1953), as well in conditions involving either object motion or self-motion (Wallach, Stanton, \& Becker, 1974; Rogers \& Graham, 1979, 1982).

It should be noted that the concept of a perceptual invariant as characterized in the ecological approach is not limited to specific sensory modalities, as vision. Invariants can be both modal and multimodal and depend on proximal information made available to the agent via the somatosensory and vestibular systems on top of other sensory modalities. For instance, an important number of studies have tackled the issue of "dynamic touch", the ability of the agent to extract invariant properties of rotational dynamics (the inertia tensor), which may specify objects and body properties (Carello et al., 2006; Carello \& Turvey, 2000; Turvey, 1996).

\footnotetext{
${ }^{7}$ It should be noted that merely extracting invariant cross ratios cannot explain the perception of rigidity in complex cases, for which further perceptual information is required (Koenderink, 1986).

${ }^{8}$ These invariants are referred to as "first-order differential invariants" of optic flow because their values are independent of both the choice of the coordinate system and any rotations of the observer around the projection center. As Koenderink (1986) clarifies: "The [divergence] is a number that specifies the relative time change of apparent area (solid angle) of a piece of the optic array, the curl is a number that specifies the rate of rotation, and the [deformation] can be specified with a number (the degree of shear: always positive) and an orientation (the axis of contraction)".
} 
The above examples illustrate the idea according to which perceptual capacities rely on invariants that the agent can extract from a multimodal array of sensory information when this sensory information is modulated by motor behavior. To summarize, we can say that in the ecological approach, a perceptual invariant is a property of the proximal sensory array that remains unchanged throughout motor interaction with the environment. It is action-dependent insofar as it can only be revealed when the sensory stimulation undergoes transformation, which is typically the result of motor behavior.

\subsection{Sensorimotor invariants}

The idea that the joint effects of self-initiated movement and sensory stimulation can provide information to perceptual systems is the core assumption of sensorimotor approaches to perception. Sensorimotor invariants-often referred to as "sensorimotor contingencies" (MacKay, 1986; O’Regan \& Noë, cit.) or “sensorimotor dependencies" (Broackes, 2001; Philipona et al. 2003) —are the basic constituents of actiondependent perceptual information in sensorimotor theories. O'Regan and Noë (cit.), propose the following informal characterization of sensorimotor contingencies:

$[t]$ he structure of the rules governing the sensory changes produced by various motor actions, that is, what we call the sensorimotor contingencies governing visual exploration (p.941).

This definition is quite loose, and in any case not sufficiently constrained to let us appreciate any significant difference between invariants studied from a sensorimotor or ecological perspective. A better characterization of a sensorimotor invariant can be found in the formal definition of "sensorimotor laws" proposed by Philipona et al. (2004). Their goal is to characterize the invariance governing relations between motor efference $(M)$ and sensory reafference $(S)$. Given the configuration of the body (referred to as $P$ ) and that of the environment $(E)$, they propose that:

- $\quad P$ and $M$ are connected through a function $\varphi_{a}: P=\varphi_{a}(M)$

- $S$ is connected to $E$ in virtue of a function $\varphi b: S=\varphi b(P, E)$

The following relation is then introduced as a functional sensorimotor law:

$$
\varphi(M, E)=\varphi b(\varphi a(M), E)
$$

Although this formal characterization can be challenged on some of its underlying assumptions ${ }^{9}$, it is sufficiently explicit to contrast the notion of a sensorimotor invariant from that of an ecological invariant. Sensorimotor laws constrain the way in which motor efference and sensory reafference systematically co-vary, as a function of both the configuration of the body and the structure of the environment. In a given environment, the body configuration is controlled by the motor outputs in virtue of $\varphi_{a}$. The sensory organs altogether deliver a multidimensional input that is, in turn, a function $\varphi_{b}$ of the configuration of the body and the configuration of the environment.

\footnotetext{
${ }^{9}$ For instance, body configurations need not be uniquely a function of motor states but may also depend on environmental states (e.g. orientation with respect to gravity).
} 
Given the existence of these nomological relations in the agent-environment system, sensorimotor approaches assume that perceptual systems are able to extract invariant relations between co-occurring sensory and motor patterns. Sensorimotor invariants are by hypothesis the basic constituents of any kind of information perceptual systems are able to parse when an organism interacts with the environment. In this sense, sensorimotor theories endorse the controversial view that all perceptual information is inherently efference-dependent.

Given these considerations, we can now formulate a sufficiently distinctive characterization of an action-dependent perceptual invariant from a sensorimotor perspective. In contrast to ecological approaches, sensorimotor theories do not characterize perceptual invariants as properties of the sensory stimulation revealed through motion. A perceptual invariant in a sensorimotor approach is a property of sensorimotor couplings that remains invariant throughout transformations. Whenever a sensory transformation co-occurs with a movement, those properties that remain unchanged in this coupling constitute a sensorimotor invariant. Sensorimotor invariants are then action-dependent insofar as they describe sensory transformations matching specific movements and, vice versa, motor transformations matching specific sensory changes.

\subsection{Ecological vs. sensorimotor invariants}

As noted in the beginning of our analysis, the radical character of sensorimotor and ecological approaches does not consist in the claim that action is required in the perception of one's own movement, bodily configuration or, more broadly, in perception targeted at the control of spatial behavior (which is plainly compatible with mainstream perceptual theories). Rather, the radical claim consists in saying that even the perception of (supposedly) agent-independent properties (typically: exteroception) relies on action-dependent information. Our thesis is that radical approaches diverge in their interpretation of the claim that action pervasively modulates perception, since they provide substantially different characterizations of what constitutes perceptual information. In particular, we have shown that ecological and sensorimotor approaches endorse different notions of perceptual invariants, and, more specifically, that they make contrasting assumptions on the relation of a perceptual invariant to the agent's movement.

We submit that, in an ecological framework, it is not necessary to refer to specific movements in order to characterize perceptual invariants but only to properties of sensory patterns that remain invariant across possible transformations. In contrast, in a sensorimotor framework, invariants are by definition properties of co-occurring sensory reafference and motor efference. More generally, we can say that movement has a constitutive role in the determination of sensorimotor invariants, whereas it is instrumental in the determination of ecological invariants.

Let us try to unpack this idea. From an ecological perspective, properties of the sensory stimulation that do not change under transformation are taken as perceptual invariants and as bearers of information about states of the agent-environment system, in virtue of the ecological laws. Hence, picking up such invariants enables the observer to perceive a given state, event or property related to the agent, the environment or their mutual relation. If an ecological invariant is described by specific 
transformations in the multisensory array, movements of the agent are only required to produce the sensory transformation with respect to which the invariant is defined. However, in terms of ecological laws, multiple movements can in principle produce identical transformations and reveal the same invariants. We then conclude that ecological invariants are defined in the general case with respect to a specific transformation, but not to the movements that produce these transformations.

It should be emphasized that the instrumental role of movement in constituting ecological invariants is not at odds with the fact that they can provide information about the agent's body configuration and movements when needed. Perceptual invariants encompassing, for instance, somatosensory and kinesthetic stimulation can specify proprioceptive exproprioceptive and/or exteroceptive information (the literature about dynamic touch we mentioned before is particularly relevant here). However, from the fact that these sensory sources can provide agent-related information, it does not follow that the underlying invariants encode movements (and, in particular, efference) as constitutive variables ${ }^{10}$.

Consistently with the idea of the instrumental role of movement, the ecological approach admits the possibility that, in many perceptual conditions where information about the agent's movements or body configuration is not necessary (typically, in cases of exteroceptive perception), invariants not encompassing agentrelated variables could provide sufficient information to accomplish the perceptual task. As we will clarify in the following sections, distinguishing between the constitutive role of movement in invariants and the fact that sensory invariants can inform the agent about its own movements has crucial implications on the explanatory strategy of the two approaches.

The idea of the instrumental role of movement in ecological approaches can be exemplified by considering the two cases of perceptual invariants described above.

The cross ratio is invariant under different transformations affecting either the viewpoint $\mathrm{X}$ or the object in the environment (L1). This means that, in real-world situations, the same invariant would be revealed either by active movements of the perceiver around a rigid stationary object or by a moving object for a static observer. Even if we consider active movements of the perceiver, the same invariant can be revealed either by the active manipulation of the object by the perceiver or by an active displacement of his viewpoint. Since what matters from an ecological perspective is the informative relation between the perceptual invariant (e.g., the cross ratio) and the specified property of the distal object (e.g., rigidity), the specific motor patterns producing the sensory transformation do not contribute to the characterization of the invariant.

Similarly, what specifies motion parallax as an invariant is a transformation that underdetermines the class of possible movements that produce it. In order for parallax to be revealed, the relevant sensory transformation has to occur, but it makes no difference, in terms of the availability of the information, if the invariant is revealed by an active movement of the observer, by a passive movement of the observer, or by a movement of the object in the environment.

\footnotetext{
${ }^{10}$ Incidentally, different sources of motor-dependent information can fail to uniquely specify the state of the agent, see for example the discussion about input conflicts in Stoffregen \& Bardy (2001).
} 
In contrast, according to a sensorimotor approach, when actively exploring the environment an organism is exposed to dynamic sensorimotor couplings, which possess properties invariant under transformations affecting various efferent motor and reafferent sensory variables. For instance, invariant relations can be described between a rotation of the head on the one hand and the co-occurring patterns of retinal and proprioceptive stimulation on the other hand. These sensorimotor relations remain invariant under transformations affecting sensory or motor parameters (i.e., changes in rotation velocity, changes in initial conditions, variations in stimulus configuration, or intensity). The perceiver is able by hypothesis to identify sensorimotor invariants over several occurrences of sensorimotor couplings.

We can summarize these conclusions by saying that these two characterizations of the notion of action-dependent perceptual invariants put different constraints on the kind of information an organism is supposedly able to extract and to process. In the case of sensorimotor approaches, the organism is supposed to be able of extracting motor-specific information whereas in the case of ecological theories the organism must be able to extract transformation-specific information.

\subsection{Motor dependence vs. action dependence}

Are we entitled to conclude that ecological invariants, given their instrumental relation with movement, are not action-dependent? This claim may sound counterintuitive, since the fundamental assumption of the ecological approach is admittedly that perceptual information is only available to agents actively exploring their environment. Perceptual information is available to agents in terms of affordances, i.e. it specifies "possibilities for action" (Gibson, cit.; Withagen \& Michaels, 2004). Invariants specify information only to animals engaging in actions and in this sense information provided by invariants has been considered as constitutively action-dependent (Gibson, 1979; Reed, 1996; Warren, 2006b). As Michaels and Carello (cit.) observe:

[e]nergy patterns that are invariant with respect to relevant transformations and that specify the environment are not, by themselves, equivalent to information; the animal must be specified. Moreover, even a complete inventory of those invariant structures must be based on an equally complete inventory of the consequences of each of the exploratory (and performatory) activities in which an animal can engage (p.39).

How can we accommodate our claim of the instrumental role of motion in ecological approaches with the claim that action is constitutive of perceptual information according to defendants of ecological theories? The solution, we argue, is that in the ecological approach the term "action" refers to adaptive, goal-directed behavior (Gibson, cit.; Reed, cit.). By picking up perceptual invariants, organisms perceive affordances that mediate adaptive control of behavior. In ecological terms then, the radical claim that information is "constitutively action-dependent" means that animals perceive affordances in the environment that allow them to attain specific goals. ${ }^{11}$

\footnotetext{
${ }^{11}$ As Warren (2006b) puts it: “Alongside the dynamical approach to movement there developed the ecological perception-action approach to the control of behavior (Gibson, 1958/1998, 1979; Lee, 1976, 1980; Shaw, Kugler, \& Kinsella-Shaw, 1990; Turvey \& Carello, 1986; Warren, 1988, 1998).
} 
Information in terms of affordances is in this sense targeted to specific actions, but the invariants (whose pickup reveals an affordance) do not depend, as we argued, on specific movements. As Reed puts it:

[i] nformation is specific to the ecological task of the animal. It is not specific to mechanisms or to processes within the animal, nor is it purely external unrelated to the organism (Reed, 1996, p. 57). ${ }^{12}$

Ecological approaches can then at the same time defend a broad idea of action dependence of information (i.e. its specificity to the task) without discarding the idea of the instrumentality of movement at the level of perceptual invariants. As Withagen \& Michaels (2004) point out, Gibson (1966)'s concept of a "perceptual system" as well as Reed (1982)'s concept of an "action system" assume the functional dependence of action and perception at the level of goal-directed behavior. The same information can be "captured" by different perceptual systems which may physically vary, and cannot be described with reference to specific anatomical structures (Reed, 1986).

The instrumental role of movement in the definition of invariants appears to be perfectly consistent with this theoretical stance: ecological psychologists refer to this idea as "motor equivalence". The ecological approach insists on the idea that perceptual systems are able to exploit information which is invariant over variations of body configuration, movements and even anatomical structure. This capacity has an adaptive value, to the extent that it enables agents to obtain equivalent information under different conditions. Again, emphasizing the idea of motor equivalence does not conflict with the fact that ambient arrays may provide invariants specifying proprioceptive and exteroceptive information. Rather, it implies that agent-related information will only be used when relevant with respect to the perceptual task, but neglected when irrelevant, consistently with the idea of the adaptive value of motor equivalence.

To conclude, if we take action to mean "goal-directed motor behavior", then we can say that the sensorimotor approach shares with the ecological approach the assumption that information is action-dependent in a loose sense. ${ }^{13}$ However, we argued that sensorimotor and ecological theories make contrasting assumptions on the nature of perceptual invariants that constitute information for the agent. Albeit loosely "action-dependent" in both cases, we suggested that perceptual information is characterized as motor-specific in the case of sensorimotor invariants and transformation-specific (and motor-equivalent with respect to movement) in the case of

\footnotetext{
This view emphasizes the role of occurrent information in guiding behavior, in the form of optic, acoustic, haptic, or olfactory fields that are structured by and are specific to the state of the agentenvironment system. The research program involves determining what informational quantities govern naturalistic behaviors like reaching, catching, hitting, standing posture, or locomotion".

${ }^{12}$ By taking the classical example of $\tau$ (Lee, 1980), Reed explains that "The hummingbird's ability to maintain $\tau>0.5$ is an ability to dock with a slight closing velocity. Bats also seem to control locomotion by monitoring $\tau$. Despite the radical difference in mechanisms (vision versus echolocation, bird versus bat flight patterns), these animals are accomplishing the same thing" (Reed, 1996, page 57).

13 "Enactive knowledge depends upon an action-perception cycle. Action reveals information, which guides further action, which reveals additional information, and so on" (Stoffregen \& Bardy, 2004).
} 
ecological invariants.

This distinction between action dependence and motor dependence has been underestimated so far in the literature. Part of the major theoretical divergence between enactive and ecological theories (in particular their different view of agents as shaping the perceptual environment through action vs. agents simply exploring the perceptual environment through action) should actually be reconsidered in the light of this technical distinction about the underlying notion of perceptual information. ${ }^{14}$

We submit, though, that this distinction is useful not only to distinguish the ecological and sensorimotor approach at a theoretical level, but also to disentangle different empirical predictions that depend on this distinction and that the literature has not yet fully acknowledged.

\section{Motor specificity: empirical predictions of the sensorimotor hypothesis}

If - as sensorimotor theories assume-perception is motor-dependent insofar as it systematically relies on information that ties sensory reafference to specific classes of motor efference (and not only to motor-induced sensory transformations), then we should expect a substantial divergence in the empirical predictions made by ecological and sensorimotor theories.

The question of the empirical validation of these approaches reveals a major asymmetry between the sensorimotor and the ecological theory. On the one hand, the ecological approach to perception can be seen as a full-fledged scientific theory, which has received in four decades of investigations substantial empirical support ${ }^{15}$. Ecological psychologists have developed detailed descriptions of how perceptual systems exploit different sources of information available in the ambient arrays for the adaptive control of behavior. On the other hand, as we already pointed out, the sensorimotor approach has not yet been translated into an empirical research programme, and has been criticized precisely for the lack of empirical evidence in support of its core assumption (Scholl \& Simons, 2001). In particular, the thesis of the constitutive and pervasive role of movement in perception appears to be an extremely strong claim, which, as many have suggested, might be trivially refuted by cases in which accurate perceptual performance can occur with no motor contribution of the agent (Prinz, 2006). The ecological perspective, by putting forward the idea that perceptual information is motor equivalent, seems to be offer a more plausible account of how to characterize the relations between action and perception.

Moreover, one may argue that, even if the theoretical distinction between the constitutive and instrumental role of movement in defining perceptual invariants holds, this makes virtually no difference between the two approaches, to the extent that "constitutively" motor-equivalent invariants may specify, when needed, agentrelated (e.g. proprioceptive and exproprioceptive) information. Hence, the ecological approach can account for all perceptual tasks that require a reference to the agent's

\footnotetext{
14 See also Varela et al., 1991, pp. 203-204 for a similar conclusion.

${ }^{15}$ A representative bibliography of the theoretical and experimental work produced by the ecological approach can be found here: http://ione.psy.uconn.edu/publications.html
} 
self-motion, without postulating the pervasiveness of efference-dependent information.

In this section, we address the issue of the empirical basis by reviewing evidence from the experimental literature in which encoding motion specificity seems to be a key to the explanation of a number of perceptual capabilities. The logic of this section consists in showing that there seems to be cases in which motor specificity plays a role in modulating perceptual judgments even if it does not provide additional information which is relevant for the task. In other words, there are conditions in which efferencespecific information seems to be required not only to perform proprioceptive and exproprioceptive tasks targeted at the control of motor behavior (in which case the ecological and sensorimotor approach would make similar predictions), but also in the case of exteroceptive judgments where movement should not in principle contribute to further specify the property or state to be perceived. These examples are not meant to be a rebuttal of the ecological thesis of the functional equivalence of motor mechanisms, which has already received substantial empirical support (see section 2.1 above). Rather, our aim is to suggest that the sensorimotor notion of motor specificity should be carefully distinguished from that of motor specificity since the former could be in a better position than the latter to account for some specific classes of perceptual abilities.

\subsection{Active vs. passive perception}

The first set of experimental results we review are those contrasting the performance of immobile vs. moving observers when they engage in perceptual tasks involving identical sensory configurations.

\section{Perceptual substitution}

Perceptual substitution is a manipulation that allows to transform stimuli in a given sensory modality (e.g. vision) into stimuli of another sensory modality (e.g. touch). A perceptual substitution device consists of three parts: a sensor allowing the conversion of a form of energy (stimuli from sensory modality A) into signals interpretable by a coupling system, responsible of the coordinated activation of a stimulator into sensory modality B. The goal of protocols using perceptual substitution devices consists in measuring to what extent perceptual systems are able to correctly process information independently of the modal coding in which the senses present it.

Experimental results obtained by perceptual substitution protocols (Bach-y-Rita, 1972; Barfield \& Furness, 1995; Lenay, 1997; Gonzales \& Bach-y-Rita, 2003) support the claim that perceptual processing required to solve these tasks rely on the availability of efferent information. Particular emphasis has been put on the fact that efficient perceptual substitution only occurs if subjects are allowed to engage in sensorimotor interaction with the object, whereas no perception arises if dynamical sensory stimulation is passively received and processed by the subject (White, 1970; Lenay 2003; Sampaio, 1995). The interesting point is that, when subjects are allowed to actively interact with the object, in many cases motor patterns do not reveal subject-related sensory stimulation, which would provide additional relevant information for accomplishing the task. To consistently account for these cases, the ecological approach should show that 1) only the active conditions provide adequate 
information to the subject and 2) in the active conditions there is motor equivalence ${ }^{16}$. Otherwise, from an ecological perspective one could expect a similar performance in both cases, since the relevant invariants produced during the transformation of the stimuli are supposedly the same in the active and passive conditions. In contrast, the sensorimotor approach straightforwardly predicts a different performance in the two conditions, assuming that the observer, in the passive condition is not engaging in any kind of sensorimotor coupling, and as a consequence no perceptual invariants can be extracted in this condition.

\section{Structure-from-motion}

In contrast with the results discussed in section 2.1, which support the hypothesis of motor equivalence, several studies have shown that subjects process identical visual configurations in different ways, depending whether they actively produce the transformation in the visual stimulation or they passively process it (see Wexler \& Van Boxtel, 2005 for a comprehensive review). There is growing evidence that the perception of depth and object shape from motion parallax considerably varies depending on whether the observer is allowed to move or not (Wexler et al., 2001a; Wexler et al., 2001b; van Boxtel et al., 2003). Typical test conditions are those in which subjects are asked to disambiguate a $3 \mathrm{D}$ optic flow simulated on a computer monitor. The optic flow is presented in such a way that the same ambiguous configurations are present when subjects passively experience the stimuli or when they are allowed to move towards the object. Wexler et al. (2001a) show that subjects provide different interpretations of the stimuli in the passive and active conditions, despite the fact that they are exposed to the very same visual sensory transformations. Several hypotheses have been proposed to account for the reason why the perception of the very same visual configuration varies when the subject is moving (Wolpert $\&$ Flanagan, 2001). All of these hypotheses require that the agent be capable of predicting the correlation between specific efferent patterns and specific sensory transformations. The difference between the two conditions cannot be explained solely by referring to sensory transformations (Gibson, 1950; Findlay \& Gilchrist 2003). Again, the fact of perceiving differently an identical optic flow seems to be at odds with the ecological assumption of motor equivalence, since in this case selfmotion does not reveal additional information supposed to be relevant for the perceptual task. Conversely, if the difference in performance is due to different ways of processing sensorimotor couplings, then sensorimotor invariants seem to be the relevant structures that agents are extracting.

A defendant of the ecological approach may also reply that all these experimental protocols comparing active vs. passive conditions do not provide any evidence specifically in favor of the sensorimotor hypothesis, since the ecological approach would equally predict that, as far as in one condition the perceiver is not actively

\footnotetext{
${ }^{16}$ For instance, Warren (2006a) reports evidence that depth perception in sensory substitution is not motor specific, since it would transfer from one arm to the other as well as to novel joint angles. He suggests that this is evidence of a perception-action invariant relevant for sensory substitution that is not motor-specific.
} 
exploring the environment, his performance will trivially be different from the active condition. Passive conditions, as it were, do not provide information in terms of affordances because there is no goal-directed behavior. However, insofar as the observer can make distinct perceptual judgments, we argue that it is legitimate to consider information provided by the simulated optical flow as "affordance" in a genuine ecological meaning. The fact the perceiver is not actively moving should make no difference if motor patterns coupled with the sensory stimulation were irrelevant to the task.

\subsection{Perception in active vs. passive self-motion}

A second set of experimental results consistent with the hypothesis of motor specificity of perceptual invariants are those suggesting that subjects perceive differently identical dynamic visual configurations whether their movement is selfgenerated ("voluntary") or passively imposed. For instance, it is know for a long time that perceptual adaptation to prismatic distortions is much more efficient in conditions where movement is self-generated than in conditions where participants are passively moved on a trolley or in a wheelchair (Held, 1965). Analogously, active self-motion has been shown to improve heading judgments when compared to passive self-motion (Telford et al., 1995).

Wexler (2003) compares a control condition in which subjects experience an ambiguous dynamical configuration (which can be interpreted as stationary either in an allocentric frame of reference, or in an egocentric frame of reference) when engaging in self-generated head movements with a condition in which subjects have their head blocked and are passively displaced on a wheelchair by the experimenter. With respect to the active/passive comparison, in this case subjects are able to move their head in both conditions, which provides them with additional proprioceptive information. The results show a significant difference in the interpretation of the visual configuration in the two conditions: subjects display a systematic preference for the allocentric stationary interpretation when head movements are self-generated. Crucially, Wexler shows in the same study that perceptual performance changes not only as a function of the active or passive character of self-motion, but also of the specific kind of active movement performed by the subject. Subjects presented with an identical visual pattern are asked to perform the same task of stimulus disambiguation either by freely moving their head or by actively moving the wheelchair without the possibility of moving their head. In this case, results show that the interpretation of the visual stimuli was significantly different. Whereas subjects preferred again an allocentric interpretation when allowed to actively move their head, they systematically preferred an egocentric interpretation when forced to move the wheelchair, thus showing the determinant role of the specific motion scheme in modulating perceptual judgments.

The standard account for these results is that subjects perform differently in the two conditions because active self-motion provides additional extra-retinal information with respect to passive self-motion. In particular, efferent copies are assumed to enable sensorimotor predictions-i.e. anticipations of sensory effects resulting from specific movements-which may be used to constrain perceptual judgments through the comparison between expected reafference and actual 
sensations (Wolpert \& Flanagan, 2001; Blakemore et al., 1999). In the specific case of ambiguous configurations discussed, sensorimotor prediction may be used to constrain the possible interpretations of the visual configuration (Wexler \& Van Boxtel, 2005). As Wexler emphasizes, the hypothesis of sensorimotor predictions is consistent with the fact that the perceptual performance varies following the specific motor scheme in which the subject is engaged.

Evidence on the contribution of voluntary self-motion to perception represents a second example of empirical results supporting the idea that ecological invariants that postulate motor dependence cannot per se account for cases in which different motion patterns coupled with the same sensory stimulation result in difference in performance. Sensorimotor theories would argue that the difference in the two conditions is due to the use of distinct types of underlying motor-specific invariants, whereas ecological theories would need to justify the appeal to proprioceptive information as a necessary integration to disambiguate identical transformational invariants.

\subsection{Can motor-specific perceptual invariants shed light on action/perception relations?}

The results shortly reviewed in this section suggest that in several conditions only by assuming that subjects are able to parse the specific movements in which they engage when involved in a perceptual task, it is possible to adequately account for major differences in perceptual performance. Whether motor specificity can be extracted simply in virtue of underlying invariants or requires the integration of a proprioceptive judgment based on non motor-specific information is an issue that require further empirical investigation. These cases are meant to illustrate that theories endorsing different notion of action-dependent perceptual invariants can be contrasted not only at a conceptual level but also in terms of the different empirical predictions they formulate on specific perceptual tasks. Of course, these results do not provide compelling evidence that perceptual systems, when negotiating stimulus interpretation through action, always rely on motor-specific strategies. Quite on the contrary, the ecological hypothesis of functional equivalence of motor patterns is well established and has received broad empirical support, which is in fact at odds with the predictions of sensorimotor theories. What this empirical evidence suggests is that the question of understanding to what extent action modulates perceptual processing cannot be settled without a thorough understanding of the structure of perceptual information mediated by action. For instance, an advocate of the ecological approach may argue that these results simply suggest that, as far as the agent behaves in more "ecologically-valid" conditions, agent-related information provided by self-motion can contribute to exteroception in a broad range of conditions. However, it seems that this general interpretation would significantly weaken (if not undermine) the importance of the functional equivalence hypothesis, which is a distinctive and wellestablished feature of perceptual systems according to the ecological approach.

\section{Conclusions}

The aim of the present analysis was to frame the notion of action-dependent perceptual invariants - a notion that is central to controversial claims of the 
pervasiveness of action in the functioning of human perception. By taking a radical stance on the functional role of action in perception, ecological and sensorimotor theories are often considered as similar and mutually reducible theories. We argued that, in spite of a number of shared background assumptions, ecological and sensorimotor approaches to perception make substantially different predictions on the nature of perceptual information.

We showed in particular that ecological theories conceive invariants as properties of the sensory stimulation that remain constant through transformation produced by the perceiver's self-motion, but in which the actual movement generating the transformation is extrinsic to the characterization of the invariant. Ecological invariants are transformation-specific, but they do not rely on the extraction of specific motor schemes: whatever motor scheme produces a transformation in the ambient array, only the transformation is relevant to specify the perceptual invariant. In contrast, sensorimotor approaches postulate that perceptual systems build invariants by extracting regularities from co-occurring efferent motor and reafferent sensory patterns. In this case, the perceptual system is supposed to be able to represent invariances that are motor-specific, insofar as properties of motor schemes are intrinsic constituents of perceptual invariants.

This distinction allows us to drive a wedge between theories that attribute an instrumental role to self-motion in the specification of invariants and theories taking self-motion as constitutive to the specification of an invariant. Far from being a purely conceptual distinction, we suggested that this difference results in diverging empirical predictions about perceptual capabilities mediated by action. Whether an observer is extracting regularities of her own motor schemes, has substantial consequences on how perceptual judgments are affected by motor information.

Advocates of the ecological and sensorimotor approaches may still object that there is no conceptual incompatibility between the hypotheses of motor equivalence and motor specificity. Hence, each approach could easily accommodate both hypotheses in its own framework. The ecological approach could (a) include efferent information in the characterization of the global energetic array or (b) appeal to the contribution of proprioception to account for cases in which simple transformational invariants are insufficient to ground perceptual distinctions. Conversely, the sensorimotor approach may admit that (a) certain kinds of perceptual abilities need just rely on transformation-dependent information or it may even accept that (2) the organism must abstract from motor specificity for certain classes of perceptual judgments to be possible. In this case the ecological and the sensorimotor approaches would effectively reduce to the same theory. Nevertheless, such unification does not come for free. If action dependence in perception can alternatively be motor-equivalent or motorspecific, the theory will need to provide predictions on those conditions in which action modulates perception in a motor-specific way (e.g. by relying on efferencerelated information) as opposed to conditions in which this modulation is transformation-specific. Otherwise, the resulting theory, by simply encompassing two alternative hypotheses, would loose the predictive power (and empirical refutability) of the original theories.

In conclusion, there is relevant empirical support to the thesis that several perceptual capabilities are modulated by action-dependent information, as predicted 
by defendants of radical theories of action/perception relations. We suggested that further research directions will need to provide more constrained hypotheses on what it means for perceptual information to be action-dependent. This requires in particular accounting for conditions in which action modulates perception in a motorspecific way as opposed to conditions in which this modulation is transformationspecific.

\section{References}

1. Bach y Rita, P. (1972). Brain mechanisms in sensory substitution. New York : Academic Press.

2. Barfield, W., \& Furness, T.A. (1995). Virtual environments and advanced interface design. Oxford: Oxford University Press.

3. Berthoz, A. (2000). The Brain's Sense of Movement. Harvard University Press.

4. Blakemore, S.J., Frith C.D., \& Wolpert, D.M. (1999). Perceptual modulation of self-produced stimuli: The role of spatio-temporal prediction. Journal of Cognitive Neuroscience, 11, 5, 551-559.

5. Broackes, J. (2001). Experience, attention, and mental representation. BBS, 224, 5, 978-979.

6. Carello, C., \& Turvey, M. T. (2000). Rotational dynamics and dynamic touch. In M. Heller (Ed.), Touch, representation, and blindness. Oxford, England: Oxford University Press, 27 - 66.

7. Carello, C., Kinsella-Shaw, J., Amazeen, E., \& Turvey, M. T. (2006). Peripheral neuropathy and object length perception by effortful (dynamic) touch: A case study. Neuroscience Letters, 405, 159-163

8. Cutting J.E. (1986). Perception With an Eye for Motion. MIT Press.

9. Duhamel, J.R., Colby, C.L., \& Goldberg. M.E. (1992). The updating of the representation of visual space in parietal cortex by intended eye movements. Science, 255, 5040, 90-92.

10. Findlay, J.M., \& Gilchrist, I.D. (2003). Active vision. The psychology of looking and seeing. Oxford : Oxford University Press.

11. Fodor, J. A., \& Pylyshyn, Z. W. (1981). How direct is visual perception? Some reflections on Gibson's "Ecological Approach". Cognition, 9,139-196.

12. Gallistel, C.R. (1980). The Organisation of Action: A New Synthesis. Hillsdale, NJ: Erlbaum.

13. Gibson J.J. (1950). The perception of the visual worl., Westport, Conn.: Greenwood Press.

14. Gibson, J. J. (1959). Perception as a function of stimulation. In S. Koch (Ed.), Psychology. A study of a science, Vol. 1, pp. 456-501. New York: McGraw-Hill.

15. Gibson, J. J. (1960). Pictures, Perspective, and Perception. Daedalus, 89, 216-27.

16. Gibson, J. J. (1966). The senses considered as perceptual systems. Boston: Houghton Mifflin.

17. Gibson, J. J. (1979). The Ecological Approach to Visual Perception. Boston: Houghton Mifflin.

18. Gibson, J. J. (1998). Visually controlled locomotion and visual orientation in animals. Ecological Psychology, 10, 161-176. (Original work published 1958).

19. Gonzalez, J.C., \& Bach-y-Rita, P. (2003). Perceptual adaptive recalibration: tactile sensory substitution in blind subjects. Behavior and Philosophy.

20. Held, R. (1965). Plasticity in sensory-motor systems. Scientific American, 213, 5, 8494. 
21. Helmholtz, H. (1878/1977). Epistemological writings. Dordrecht/Boston: D. Reidel Publishing Company.

22. Hurley, S. (1998). Consciousness in Action. Harvard University Press.

23. Hurley, S. (2001). Perception and Action: Alternative Views. Synthese, 291, 3-40.

24. Jacob, P. (2006). Why Visual Experience is Likely to Resist Being Enacted. Psyche, 12 (1) (http://psyche.cs.monash.edu.au/symposia/noe/Jacob.pdf)

25. Jacob, P., \& Jeannerod, M. (2003). Ways of Seeing: The Scope and Limits of Visual Cognition. Oxford: Oxford University Press.

26. Jarvilehto, T. (1999). The theory of the organism-environment system: III. Role of efferent influences on receptors in the formation of knowledge. Integr. Physio.l Behav. Sci., 34, 90-100.

27. Koenderink, J.J., \& Van Doorn, A.J. (1975). Invariant properties of the motion parallax field due to the movement of rigid bodies relative to an observer. Optica Acta, 22, 773-791.

28. Koenderink, J.J. (1986). Optic flow. Vision Research, 26, 1, 161-79.

29. Lee, D.N. (1976). A theory of visual control of braking based on information about time-to-collision. Perception, 5, 437-459.

30. Lee, D. N. (1980). Visuo-motor coordination in space-time. In G. E. Stelmach, \& J. Requin, (Eds.), Tutorials in motor behavior, 281-295. Amsterdam: North-Holland.

31. Lenay C., Cannu S., \& Villon P. (1997). Technology and perception : the contribution of sensory substitution systems. Second International Conference on Cognitive Technology, Aizu, Japan, Los Alamitos, IEEE, 44-53.

32. Lenay, C., Gapenne, O., Hanneton, S., Marque, C., \& Genouëlle, C. (2003). Sensory substitution : Limits and perspectives. In Y. Hatwell, , A. Streri, \& E. Gentaz (Eds), Touch for Knowing, 275-292. Amsterdam: John Benjamins.

33. MacKay, D. (1962). Theoretical models of space perception. In C.A. Muses (Ed.), Aspects of the theory of artificial intelligence. Plenum Press.

34. MacKay, D. (1987). The Organization of Perception and Action: A Theory for Language and Other Cognitive Skills. New York: Springer-Verlag.

35. Maturana, H.R., \& Varela, F.J. (1980). Autopoiesis and Cognition: The Realization of the Living. Boston Studies in the Philosophy of Sciences, Vol. 42. Dordrecht/Boston: D. Riedel Publishing Co.

36. Michaels, C. F., \& Carello, C. (1981). Direct Perception. New Jork: Prentice-Hall, Englewood Cliffs.

37. Milner, A.D., \& Goodale, M.A. (1995). The Visual Brain in Action. Oxford: Oxford University Press.

38. Nawrot, M. (2003). Depth from motion parallax scales with eye movement gain. Journal of Vision, 3, 11, 841-851.

39. Noë, A. (2002). On what we see. Pacific Philosophical Quarterly, 83, 57-80.

40. Noë, A. (2004). Action in Perception. Cambridge, MA: MIT Press.

41. Ono, M.E., Rivest J., \& Ono, H. (1986). Depth perception as a function of motion parallax and absolute-distance information. Journal of Experimental Psychology: Human Perception and Performance, 12, 331-337.

42. O'Regan, J. K., \& Noë, A. (2001). A sensorimotor account of vision and visual consciousness. Behavioral and Brain Sciences, 24, 5, 939-1011.

43. Philipona, D., O'Regan, J. K., \& Nadal, J.-P. (2003). Is there something out there? inferring space from sensorimotor dependencies. Neural Computation, 15, 9.

44. Philipona, D., O’Regan, J. K., Nadal, J.-P., \& Coenen, O. J.-M. D. (2004). Perception of the structure of the physical world using unknown multimodal sensors and effectors. Advances in Neural Information Processing Systems, 15. 
45. Philipona, D., \& O'Regan, J.K. (2006) Color naming, unique hues and hue cancellation predicted from singularities in reflection properties. Visual Neuroscience 23, 331-339.

46. Poincaré H. (1902). La science et l'hypothèse. Paris : Flammarion.

47. Port, R.F., \& van Gelder, T. (1995). Mind as motion Cambridge, MA: MIT Press.

48. Prinz, J. (2006). Putting the Brakes on Enactive Perception. Psyche, 12, 1, (http://psyche.cs.monash.edu.au/symposia/noe/Prinz.pdf).

49. Pylyshyn, Z.W. (2001). Seeing, acting, and knowing. Behavioral and Brain Sciences, 24, 5, 999.

50. Reed, E.S. (1982). An outline of a theory of action systems. Journal of Motor Behavior, 14, 98-134.

51. Reed, E.S. (1986). Motor variability but functional specificity: Demise of the concept of motor commands. Behavioral and Brain Sciences, 9, 620-622.

52. Reed, E.S. (1996). Encountering the world: Toward an ecological psychology. New York: Oxford University Press.

53. Rogers, B., \& Graham, M. (1979). Motion parallax as an independent cue for depth perception. Perception, 8, 125-134.

54. Rogers, B., \& Graham, M. (1982). Similarities between motion parallax and stereopsis in human depth perception. Vision Research, 22, 261-270.

55. Sampaio, E. (1995). Les substitutions sensorielles adaptées aux déficits visuels importants. In A.B. Safran, \& A. Assimacopoulos (Eds.), Le déficit visuel. Des fondements neurophysiologiques à la pratique de la réadaptation. Paris : Masson.

56. Shaw, R. E., Kugler, P. N., \& Kinsella-Shaw, J. M. (1990). Reciprocities of intentional systems. In Warren R., \& Wertheim A. (Eds.), Perception and control of self-motion, 579-619. Hillsdale, NJ: Erlbaum.

57. Sheerer, E. (1984). Motor Theories of Cognitive Structure: A Historical Review. In W. Prinz, \& A. F. Sanders (Eds), Cognition and Motor Processes. Berlin: Springer Verlag.

58. Scholl, B. J., \& Simons, D. (2001). Change blindness, Gibson and the sensorimotor theory of vision. Behavioral and Brain Sciences, 24, 5, 1004-1005.

59. Stark, L., \& Bridgeman, B. (1983). Role of corollary discharge in space constancy. Perception and Psychophysics, 34, 4, 371-380.

60. Steinbach, M.J., Ono H., \& Wolf, M. (1991). Motion parallax judgments of depth as a function of the direction and type of head movement. Canadian Journal of Psychology, 45, 92-98. 
61. Stoffregen T.A.n \& Bardy B.G. (2001). On specification and the senses. Behavioral and Brain Sciences, 24, 195-213.

62. Stoffregen T.A., \& Bardy B.G. (2004). Multisensory enactive interfaces and the global array, Online Paper, (http://www.interdisciplines.org/enaction/papers/3).

63. Stoffregen, T.A., Bardy, B.G., \& Mantel, B. (2006). Affordances in the design of Enactive systems. Journal of Virtual Reality Research, 10, 4-10.

64. Telford, L., Howard, I. P., \& Ohmi, M. (1995). Heading judgments during active and passive self-motion. Experimental Brain Research, 104, 502 - 510.

65. Thelen, E., \& Smith, L. B. (1994). A dynamic systems approach to the development of cognition and action. Cambridge, Mass.: MIT Press.

66. Turvey, M.T. (1996). Dynamic touch. American Psychologist, 51, 11, 1134-1152.

67. Turvey, M. T., \& Carello, C. (1986). The ecological approach to perceiving-acting: A pictorial essay. Acta Psychologica, 63, 133-155.

68. Ujike, H., \& Ono, H. (2001). Depth thresholds of motion parallax as a function of head movement velocity. Vision Research, 41, 2835-2843.

69. Van Boxtel, J.J.A., Wexler, M., \& Wexler, M., (2003). Perception of plane orientation from self-generated and passively observed optic flow. Journal of Vision, 3, 318-332.

70. Varela, F. (1979). Principles of Biological Autonomy. New York : North Holland.

71. Varela F., Thompson E., \& Rosch E. (1991). The Embodied Mind: Cognitive Science and Human Experience. Cambridge, MA: MIT Press.

72. Wallach, H., \& O'Connell, D.N. (1953). The kinetic depth effect, Journal of Experimental Psychology, 45, 205-217.

73. Wallach, H. Stanton, L., \& Becker, D. (1974). The compensation for movementproduced changes in object orientation. Perception \& Psychophysics, 15, 339-343.

74. Warren, W. H. (1988). Action modes and laws of control for the visual guidance of action. In O. Meijer, \& K. Roth (Eds.), Movement behavior: The motor-action controversy, 339-380. Amsterdam: North-Holland.

75. Warren, W. H. (1998). Visually controlled locomotion: 40 years later. Ecological Psychology, 10, 177-219.

76. Warren W.H. (2006a). Enactive Knowledge: Sensory-motor expectancies or perception-action invariants?. Invited talk at the Enactive/06 Conference, Montpellier.

77. Warren, W.H. (2006b). The Dynamics of Perception and Action. Psychological Review, 113, 2, 358-389. 
78. Wexler, M., Panerai, F. Lamouret, I., \& Wexler, M., (2001a). Self-motion and the perception of stationary objects. Nature, 409, 85-88.

79. Wexler, M., Lamouret, I., \& Droulez J. (2001b). The stationarity hypothesis: an allocentric criterion in visual perception. Vision Research, 41, 3023-3037.

80. Wexler, M. (2003). Allocentric perception of space and voluntary head movement. Psychological Science, 14, 340-346.

81. Wexler, M.J, \& Van Boxtel, J.J.A. (2005). Depth perception by the active observer.Trends in Cognitive Sciences, 9, 431- 438.

82. White, B.W., Saunders, F.A., Scadden, L., Bach y Rita, P., \& Collins, C.C. (1970). Seeing with the skin, Perception and Psychophysics, 7, 23-27.

83. Withagen, R., \& Michaels, C.F. (2005). On Ecological Conceptualizations of Perceptual Systems and Action Systems. Theory \& Psychology, 15, 5, 603-620.

84. Wolff, P. (2004). Position of code and code for position: From isomorphism to a sensorimotor account of space perception. Visual Cognition, 11, 137-160.

85. Wolpert, D.M., \& Flanagan, J.R. (2001). Motor prediction. Curr. Biol., 11, 729-732. 